\title{
Diamagnetism and flux creep in bilayer exciton superfluids
}

\author{
P. R. Eastham, ${ }^{1}$ N. R. Cooper, ${ }^{2}$ and D. K. K. Lee $^{3}$ \\ ${ }^{1}$ School of Physics, Trinity College, Dublin 2, Ireland \\ ${ }^{2}$ T. C. M. Group, Cavendish Laboratory, J. J. Thomson Avenue, Cambridge CB3 OHE, United Kingdom \\ ${ }^{3}$ Blackett Laboratory, Imperial College London, London SW7 2AZ, United Kingdom
}

(Received 20 December 2011; revised manuscript received 20 March 2012; published 25 April 2012)

\begin{abstract}
We discuss the diamagnetism induced in an isolated quantum Hall bilayer with total filling factor $v_{T}=1$ by an in-plane magnetic field. This is a signature of counterflow superfluidity in these systems. We calculate magnetically induced currents in the presence of pinned vortices nucleated by charge disorder, and we predict a history-dependent diamagnetism that could persist on laboratory time scales. For current samples, we find that the maximum in-plane moment is small, but with stronger tunneling the moments would be measurable using torque magnetometry. Such experiments would allow the persistent currents of a counterflow superfluid to be observed in an electrically isolated bilayer.
\end{abstract}

DOI: 10.1103/PhysRevB.85.165320

PACS number(s): 73.43.Nq, 73.43.Jn, 73.43.Lp

\section{INTRODUCTION}

Superfluidity $^{1}$ is a spectacular form of hydrodynamics involving dissipationless flow, metastable circulation, and quantization of circulation. It occurs in liquid helium and cold atomic gases, where it is associated with the condensation of many bosons into a single quantum state. Whether condensates of quasiparticles, such as excitons, would also be superfluids has been discussed for many years, with debate over the physical manifestations ${ }^{2-8}$ of superfluid hydrodynamics for quasiparticles, the (related) role of symmetry-breaking perturbations, and the significance of interactions and thermal equilibrium. ${ }^{9}$

This question can now be addressed experimentally, with emerging evidence for the condensation of quasiparticles including excitons, polaritons, and magnons. A particularly interesting system is the quantum Hall bilayer at total filling factor $v_{T}=1$ (see Fig. 1). This consists of two closely spaced quantum wells, each containing a two-dimensional electron gas, subjected to a strong perpendicular field $B_{\perp}$ so that the lowest Landau level in each layer is half-filled. The tunneling between the two layers is weak compared to the Coulomb energy scale. When the interlayer separation $d$ is of the order of the magnetic length $l_{B}=\left(\hbar / e B_{\perp}\right)^{1 / 2}$, the ground state is believed to be a Bose-Einstein condensate of interlayer excitons ${ }^{10-15}$ caused by the Coulomb attraction of electrons and holes across the layers. (Note that the holes are unfilled electron states of the lowest Landau level of the conduction band.) Flows of these condensed excitons correspond to dissipationless counterflowing electrical currents in the two layers. ${ }^{12}$ Initial evidence of dissipationless transport came from interlayer tunneling, ${ }^{16,17}$ which exhibits nonzero interlayer currents at negligible interlayer voltages. ${ }^{12,18-20}$ This regime persists up to a critical current, above which dissipation increases rapidly.

The interpretation of transport measurements as evidence ${ }^{21}$ for exciton superfluidity is complicated by parallel chargetransport channels, the injection and removal of electrons ${ }^{7,22,23}$ at the contacts, as well as possible dissipation in the leads. Here, we show that magnetometry on isolated bilayers could provide direct evidence for exciton superfluidity, without the complications inherent in transport studies. We predict that, at low temperatures, the bilayer shows a history-dependent susceptibility. Changes in the in-plane field lead to a persistent diamagnetic moment that is, however, not induced by fields present when the condensate forms. This discrepancy corresponds to that between the moments of inertia of normal and superfluid helium inferred in a torsional oscillator experiment.

The in-plane magnetic susceptibility of the exciton condensate has previously been considered ${ }^{6,24-26}$ in clean bilayers. However, there are very good reasons to expect ${ }^{27-30}$ that disorder is essential for understanding the observed transport properties of the bilayer at low temperatures. In particular, for quantum Hall bilayers, charge disorder nucleates vortices in the condensate..$^{29-33}$ Therefore, we will study in this work the magnetic properties of a quantum Hall bilayer with disorderinduced vortices. We first consider the zero-temperature limit, and we show how dissipationless supercurrents lead to longlived diamagnetic moments. In current samples, the resulting moments are small [Eq. (16)], but for samples with stronger tunneling the moments can be comparable to the Landau diamagnetism. We also consider the effects of nonzero temperatures, and we estimate the dissipation due to thermal creep ${ }^{34}$ of the in-plane flux. This dissipative mechanism gives rise to a nonvanishing resistance for the counterflow supercurrents and causes the diamagnetic moments to decay in time. Based on the parameters of current experiments, we expect dissipation to be significant, which is consistent with experimental resistance measurements. The flux motion in this regime can be studied in transport experiments. Samples deeper in the condensed phase, however, would show persistent counterflow supercurrents on laboratory time scales, whose presence and eventual decay could be observed by magnetometry.

The remainder of this paper is structured as follows. In Sec. II, we outline the model we consider for the behavior of long-wavelength counterflow supercurrents in the disordered bilayer. In Sec. III, we apply this model to calculate the in-plane susceptibility of the bilayer in the zero-temperature limit. In Sec. IV, we discuss the extension to finite but low temperatures. Finally, in Secs. V and VI, we present numerical estimates for the moments and resistance in experiments, and we summarize our conclusions. 


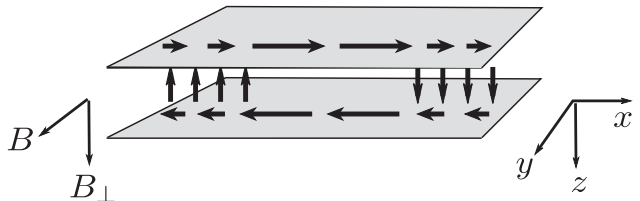

FIG. 1. Schematic diagram of a bilayer of electrons in the quantum Hall regime due to a strong perpendicular field $B_{\perp}$. Applying a weak in-plane field $B$ causes counterflowing charge currents as a diamagnetic response. For an isolated bilayer, the current loop closes at the edges of the sample by interlayer tunneling. In an excitonic picture, this corresponds to the flow of neutral excitons along the bilayer with recombination at the edges.

\section{MODEL AND BACKGROUND}

In this work, we consider a quantum Hall bilayer at total filling factor $v_{T}=1$, in the presence of the disordered electrostatic potential originating from the dopants, set back at a distance $d_{d}$. For definiteness, we adopt the "coherence network" picture of Fertig and Murthy, ${ }^{30}$ although our theory here is more general. In this picture, the random Coulomb field from the dopants creates puddles of normal electron liquid surrounded by channels of excitonic superfluid. In quantum Hall ferromagnets, physical and topological charge are related so that the charge puddles nucleate vortices, known as merons, in the superfluid channels. ${ }^{29-33}$ We estimated ${ }^{31}$ that typical disorder strengths induce on the order of one unpaired vortex per puddle so that the correlation length of this disorder is $\xi \approx d_{d} \approx 100 \mathrm{~nm}$.

The energy associated with the excitonic supercurrents in the channels is, in terms of a superfluid phase $\eta,{ }^{28}$

$$
H_{\mathrm{eff}}=\int\left[\frac{\rho_{s}}{2}|\nabla \eta+\boldsymbol{q}|^{2}-t \cos \left(\eta+\theta_{0}\right)\right] d^{2} r,
$$

where the first term is the kinetic energy of the counterflow supercurrent with superfluid stiffness $\rho_{s}$, and the second term is the energy of the interlayer tunneling currents with tunneling strength $t$. The merons introduced by the charge disorder give rise to the random field $\theta_{0}$. For the coherence network, both $\rho_{s}$ and $t$ should be renormalized by the area fraction of the superfluid channels.

Equation (1) is written in a gauge where the vector potential of the in-plane field $B$ is zero perpendicular to the layers and nonzero parallel to the layers. This field then appears in the kinetic energy, inducing a wave vector $6,12,35$

$$
\boldsymbol{q}=(\boldsymbol{B} \times \hat{\boldsymbol{z}}) e d / \hbar,
$$

where $d$ is the interlayer separation. The counterflow and tunneling currents are seen to be

$$
\begin{gathered}
\boldsymbol{j}_{\mathrm{cf}}=\frac{e \rho_{s}}{\hbar}(\nabla \eta+\boldsymbol{q})=\boldsymbol{j}_{\mathrm{p}}+\boldsymbol{j}_{\mathrm{d}}, \\
j_{t}=\frac{e t}{\hbar} \sin \left(\eta+\theta_{0}\right),
\end{gathered}
$$

so the magnetic field induces a diamagnetic contribution $\boldsymbol{j}_{\mathrm{d}}=$ $e \rho_{s} \boldsymbol{q} / \hbar$ to the counterflow supercurrent $\boldsymbol{j}_{\mathrm{cf}}$, but does not appear explicitly in the coherent tunneling current $j_{t}$. We can make a gauge transformation so that the vector potential is nonzero only perpendicular to the bilayer, in which case the phase transforms to $\eta \rightarrow \eta+\boldsymbol{q} \cdot \boldsymbol{r} \equiv \theta$ :

$$
H_{\mathrm{eff}}=\int\left[\frac{\rho_{s}}{2}|\nabla \theta|^{2}-t \cos \left(\theta-\boldsymbol{q} \cdot \boldsymbol{r}+\theta_{0}\right)\right] d^{2} r .
$$

This form for the energy functional highlights the fact that the in-plane magnetic response of the bilayer vanishes if there are no current loops that encircle the field-the field disappears from Eq. (5) when $t=0$. (A nonzero diamagnetic susceptibility remains possible in multiply connected geometries ${ }^{35}$ or at nonzero frequency. ${ }^{6}$ We comment on the extension of our results to the Corbino disk geometry in Sec. V.)

At zero temperature, the state of the bilayer is determined by minimizing Eq. (1), which gives

$$
-\lambda_{J}^{2} \nabla^{2} \eta+\sin \left(\eta+\theta_{0}\right)=0, \quad \lambda_{J}=\left(\rho_{s} / t\right)^{1 / 2} .
$$

When $\theta_{0}=0$ this is the pendulum equation, containing the Josephson length $\lambda_{J}$ : the characteristic length scale over which excitonic supercurrents decay by interlayer tunneling. This scale is estimated to be on the order of a few microns in experiments. We will discuss below the decay of excitonic supercurrents by tunneling in the disordered case, which involves a different length scale.

It is useful to recall, for comparison with our treatment of the disordered case, the response $\mathrm{e}^{24,25,36-38}$ of the clean model $\left(\theta_{0}=0\right)$ to an in-plane field. For small fields, the ground state will minimize the tunneling energy, and so $\theta \approx \boldsymbol{q} \cdot \boldsymbol{r}$ or $\eta \approx 0$. This is the commensurate state, in which the field induces a long-wavelength counterflow supercurrent $\boldsymbol{j}_{\mathrm{cf}} \approx \boldsymbol{j}_{\mathrm{d}}$, as in Eq. (3). Thus there is an in-plane magnetic moment of $M_{\|}=$ $j_{d} d L_{x} L_{y}=d L_{x} L_{y} \chi_{0} B / \mu_{0}$, with the susceptibility

$$
\chi_{0}=\mu_{0} j_{d} / B=\mu_{0} e^{2} \rho_{s} d / \hbar^{2} .
$$

We see that the diamagnetic moment $M_{\|}=\chi_{0} B$ increases linearly with the in-plane field $B$ in the commensurate state.

In an isolated bilayer, the counterflow currents must vanish at the ends of the sample. This occurs in the commensurate phase because, as dictated by Eq. (6), the diamagnetic currents in the bulk of the sample are eliminated by tunneling over a region of size $\lambda_{J}$ near the sample boundaries (Fig. 1). However, since the phase $\eta$ is a periodic variable, the maximum current which can recombine in this way is given by $e \rho_{s}|\nabla \eta| / \hbar \sim$ $e \rho_{s} / \hbar \lambda_{J}$. If the diamagnetic current present in the bulk exceeds this value, then a net winding of the phase $\eta$, in the form of Josephson vortices, enters from the boundaries. This occurs at the field

$$
B_{0} \sim \hbar / e d \lambda_{J}
$$

Above $B_{0}$, the system is in the incommensurate phase in which the kinetic energy of the counterflow supercurrents dominates so that $\theta \approx 0$ or $\eta \approx-\boldsymbol{q} \cdot \boldsymbol{r}$. The net winding in $\eta$ along the sample implies that the counterflow supercurrents have weak oscillations around zero along the sample, and the diamagnetic moment is small. The field $B_{0}$ marks the field above which the diamagnetic susceptibility rapidly decreases from $\chi_{0}$ as Josephson vortices fill the system and compensate the diamagnetic contribution in Eq. (3).

While in the clean limit the phase twists nucleated at the boundary propagate into the bulk, in the disordered case any such phase twists can be pinned by the disorder. They can thus be kept out of the bulk of the sample, which can 
continue to contribute the full diamagnetic susceptibility $\chi_{0}$. To describe this effect quantitatively, we shall use the collective pinning theory we have previously applied ${ }^{27,28}$ to the transport experiments in zero field $(q=0)$.

The starting point for understanding the transport experiments is to note that Eq. (1) is a random-field $X Y$ model. The energy $H_{\text {eff }}$ consists of the competition between the tunneling energy, which is minimized by a spatially varying superfluid phase $\eta$ over the disorder correlation length $\xi$, and the superfluid stiffness, which is minimized by a uniform superfluid phase. For the bilayer, the disorder correlation length is much shorter than the clean tunneling length: $\xi \ll \lambda_{J}$. In this regime, the superfluid stiffness dominates at short scales, up to the Imry-Ma or pinning length,

$$
L_{\mathrm{d}} \sim \lambda_{J}^{2} / \xi=\rho_{s} / t \xi
$$

where the two energies balance. We estimate ${ }^{28}$ that $L_{\mathrm{d}} \approx$ $10-100 \mu \mathrm{m}$ and $\rho_{s} \approx 20-100 \mathrm{mK}$ in experiments. Beyond this scale, the phase rotates to take advantage of the tunneling energy. Therefore, we can interpret the ground state as consisting of randomly polarized domains of size $L_{\mathrm{d}}$. The total tunneling current in each domain is zero in the ground state because the random field $\theta_{0}$ gives a current density $j_{t}$ [Eq. (4)] of random sign within each domain of constant $\eta$.

However, if we drive the system by injecting currents, phase twists enter from the contacts, leading to configurations with nonzero tunneling currents. Injected counterflow current decays into the bulk via tunneling, i.e., recombination of excitons. This tunneling current is supplied by rotating the phase of the domains near the contacts, leaving domains in the bulk in their ground state. The maximum coherent tunneling current that can be supported by each domain is given by ${ }^{28}$

$$
I_{\mathrm{d}}=e \rho_{s} / \hbar \text {. }
$$

If all the domains near the contact are rotated to supply this maximum tunneling, then we see a uniform tunneling current $j_{t}$ until all the injected counterflow current has decayed by tunneling. Then both the tunneling and counterflow currents are zero in the bulk. This can be described by the continuity equation for the current:

$$
L_{\mathrm{d}}^{2} \nabla \cdot \boldsymbol{j}_{\mathrm{cf}}= \pm I_{\mathrm{d}}
$$

where $L_{\mathrm{d}}^{2}$ is the size of the domain and $I_{\mathrm{d}}$ is the tunneling current across the layers in the domain. The sign of $I_{\mathrm{d}}$ is determined by what is necessary to reduce the counterflow current. In a one-dimensional geometry, this gives a counterflow current profile that decays linearly into the bulk from the edge. For instance, for an injected current of $-j_{i}$ at $x=0$, the current profile in the $x$ direction is

$$
j_{\mathrm{cf}}(x)= \begin{cases}-j_{i}+I_{\mathrm{d}} x / L_{\mathrm{d}}^{2}, & 0<x<x_{0}, \\ 0, & x>x_{0},\end{cases}
$$

where the point $x_{0}=j_{i} L_{\mathrm{d}}^{2} / I_{\mathrm{d}}$ marks the boundary of the region from the sample edge where coherent tunneling occurs to reduce the counterflow current. This current profile is a critical state similar to the Bean critical state in superconductors. ${ }^{39}$ In this state, there is a region near the contact over which the density gradient in the soliton train (introduced by the injected current) balances the pinning force arising from the tunneling.

\section{SUSCEPTIBILITY WITH DISORDER: ZERO-TEMPERATURE LIMIT}

We now consider the response of the disordered system to an in-plane field at zero temperature. We will see that the disordered system has a different diamagnetic response from the clean system. It does not have a commensurateincommensurate transition controlled by an intrinsic length scale $\lambda_{J}$ [see Eq. (8)]. Instead, we find a saturation phenomenon for the diamagnetic moment at a field $B_{c}$ [see Eq. (15)] controlled by the properties [Eqs. (9) and (10)] of the phase-polarized domains, i.e., the disorder pinning of the applied flux. This is followed by a depinning of the polarized domains and a strong suppression of the diamagnetic response at a higher field $B_{c 2}>B_{0}$ [Eq. (17)] controlled by the disorder correlation length $\xi$. Such behavior is similar to that of the mixed state of a superconductor, but very different from that previously predicted for exciton condensates.

We require the response of the superfluid phase $\eta$ to an in-plane field $B$, corresponding to a nonzero $\boldsymbol{q}$ in Eq. (1). To obtain this, we note that Eq. (6) for the phase $\eta$ does not depend on the in-plane field. Alternatively, we can see that the superfluid phase is coupled to the field in Eq. (1) as an integral over $\boldsymbol{q} \cdot \nabla \eta$, which can be written as a boundary term for $\eta$. Thus the current distribution in a field can be related to that in zero field with shifted boundary conditions. This observation allows us to apply the critical-state $\operatorname{model}^{27,28}$ in zero field, as reviewed above, to calculate the current profile in nonzero field.

For definiteness, we consider a rectangular sample with dimensions $L_{x}$ and $L_{y}$, with the in-plane field in the $y$ direction. Thus, counterflow currents $\boldsymbol{j}_{\mathrm{cf}}(x)$ flow in the $x$ direction, and tunneling currents $j_{t}(x)$ in the $z$ direction (see Fig. 1). For an isolated bilayer, $j_{\mathrm{cf}}(x)=0$ at $x=0$ and $L_{x}$. We can decompose $\boldsymbol{j}_{\mathrm{cf}}$ into paramagnetic and diamagnetic parts as in Eq. (3). The paramagnetic part, $\boldsymbol{j}_{\mathrm{p}}=e \rho_{s} \nabla \eta / \hbar$, obeys the boundary condition $\left.\boldsymbol{j}_{\mathrm{p}} \cdot \hat{\boldsymbol{x}}\right|_{x=0}=\left.\boldsymbol{j}_{\mathrm{p}} \cdot \hat{\boldsymbol{x}}\right|_{x=L_{x}}=-j_{d}$. Since the phase $\eta$ obeys the same equation, Eq. (6), in the bulk irrespective of an in-plane field, $\boldsymbol{j}_{\mathrm{p}}$ has the same profile as in a system in zero field (when $\boldsymbol{j}_{\mathrm{cf}}=\boldsymbol{j}_{\mathrm{p}}$ ) with a current of $-j_{d}$ flowing across the boundaries. The full counterflow current $\boldsymbol{j}_{\mathrm{cf}}$ for the isolated bilayer in a field is recovered simply by adding a uniform $+j_{d}$ to this zero-field profile of $j_{p}(x)$.

In the critical-state model as discussed above, the counterflow currents obey Eq. (11) and so $j_{p}(x)$ decays linearly in space from $-j_{d}$ at the edge to zero. In other words, the profile of the paramagnetic current $j_{p}(x)$ near the $x=0$ edge is given by Eq. (12) with $j_{i}=j_{d}$. The distance $x_{0}$ which gives the width of the region where coherent tunneling occurs is determined by the distance where the total counterflow current vanishes: $j_{p}\left(x_{0}\right)+j_{d}=0$. This criterion gives a distance of

$$
x_{0}=j_{d} L_{\mathrm{d}}^{2} / I_{\mathrm{d}}=q L_{\mathrm{d}}^{2}
$$

from the edge of the sample. The total current profile is shown in Fig. 2 (top). The figure also shows the tunneling current density $\left|j_{t}\right|=I_{\mathrm{d}} / L_{\mathrm{d}}^{2}$, which is at its maximal value up to the edge of the tunneling region at $q L_{\mathrm{d}}^{2}$ from (either) edge of the sample. Beyond $x_{0}$, there is no paramagnetic contribution to the counterflow current. 

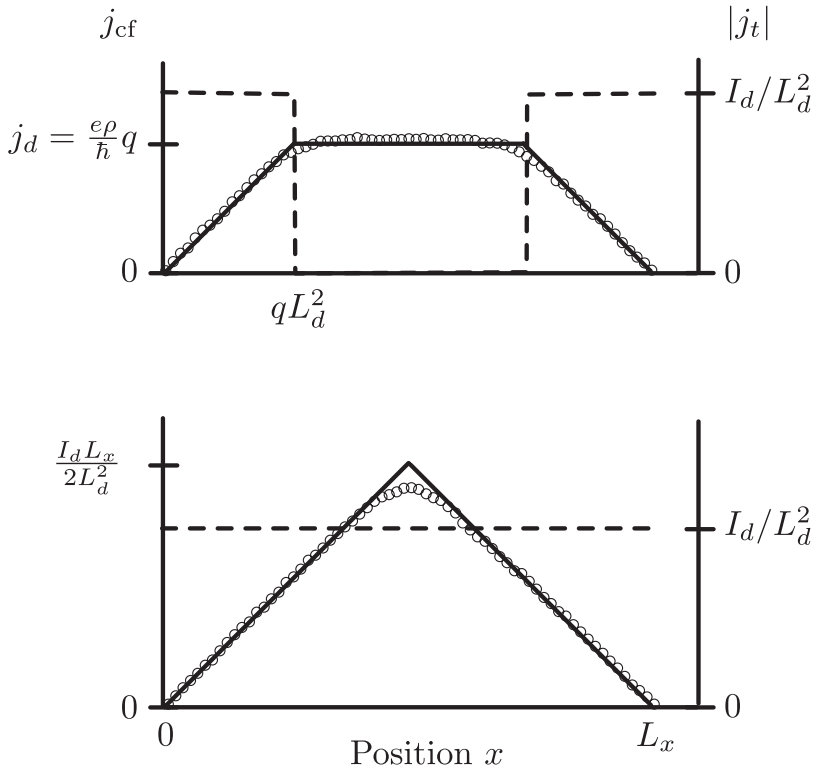

FIG. 2. Profiles of the counterflow (solid lines and circles, left axis) and tunneling (dashed, right axis) currents induced by applying an in-plane field $B$ to a rectangular bilayer (Fig. 1), at zero temperature. The lines show results from the critical-state model, and the circles show numerical results from the microscopic Eq. (6). The top (bottom) panel shows the profile below (above) the critical field $B_{c}$ [Eq. (14)]. The tunneling currents have opposite signs in the two halves of the sample (not shown). The simulation (circles) is a 200-site lattice model in one dimension with $t a^{2} / \rho_{s}=0.4$, where $a$ is the lattice spacing and $q a=6$ (top) and 12 (bottom). The disorder is uncorrelated from site to site $(\xi \approx a)$.

This current profile is similar to the one depicted in Fig. 1. A diamagnetic counterflow is generated by the in-plane field in the bulk of the bilayer. However, an isolated bilayer must have zero counterflow current at the edges. In our critical-state model, this is achieved by coherent tunneling via phase-polarized domains. The width of this tunneling region is determined by the size of the domain $L_{\mathrm{d}}$ and the coherent tunneling current that each could support $I_{\mathrm{d}}$. We see that a stronger field gives a higher diamagnetic current and so more domains must be involved. In other words, the size of the tunneling region increases with the in-plane field, as can be seen from Eq. (13): $x_{0} \propto q \propto B$. This should be contrasted with the decay length $\lambda_{J}$ for the commensurate state of the clean model, which is an intrinsic scale that does not vary with the field.

As the parallel field $B$ is increased, the diamagnetic current in the bulk increases linearly with $B$. This requires a wider tunneling region so that all the current can decay to zero at the edge. So, the tunneling region increasingly penetrates the bulk. This penetration is complete when the width of the region, $q L_{\mathrm{d}}^{2}$, reaches $L_{x} / 2$. This saturation occurs when the field reaches a critical field of

$$
B_{c}=(\hbar / 2 e)\left(L_{x} / d L_{\mathrm{d}}^{2}\right)=(\hbar / 2 e)\left(L_{x} \xi^{2} t^{2} / d \rho_{s}^{2}\right) .
$$

Beyond this critical field, all the phase domains in the sample take part in coherent tunneling with a constant magnitude for the tunneling current density $j_{t}$, as depicted in Fig. 2 (bottom). The counterflow current, therefore, rises linearly from zero from either edge, reaching a maximum at the center of the sample. This saturated current profile stays the same for fields higher than $B_{c}$. Note that, whereas the critical field $B_{0}$ in the clean limit is a microscopic parameter independent of sample geometry, $B_{c}$ is proportional to the length of the sample in a disordered system.

We have tested the critical-state model by comparing it with numerical minimization of the energy [Eq. (1)] for a one-dimensional lattice model. To do this, we add a dissipative dynamical term $-\dot{\eta}$ to the right-hand side of Eq. (6) and numerically find the resulting steady state. We begin by finding the steady state with the boundary condition $d \eta / d x=\left.0\right|_{x=0, L_{x}}$, corresponding to an isolated bilayer in the absence of an in-plane field. We then slowly increase the boundary condition so that $d \eta /\left.d x\right|_{x=0, L_{x}}=-q$, corresponding to applying the inplane field. The counterflow supercurrent is then obtained from the resulting phase profile by adding the diamagnetic term, as described above. The resulting current profiles, averaged over disorder realizations, are shown as the circles in Fig. 2, and are seen to agree closely with the critical-state model.

We will now discuss the magnetic moment generated by the diamagnetic response of the bilayer. Integrating the current profile gives the moment $M_{\|}$:

$$
\frac{\mu_{0} M_{\|}}{L_{x} L_{y} d}= \begin{cases}\chi_{0} B\left(1-\frac{B}{2 B_{c}}\right), & B<B_{c}, \\ \chi_{0} B_{c} / 2, & B \geqslant B_{c} .\end{cases}
$$

We see that the moment no longer rises linearly with $B$ with the full diamagnetic susceptibility $\chi_{0}$ of the clean system. This is because, as seen in Fig. 2, the fraction of the sample with the full diamagnetic current $j_{d}$ is continuously reduced as $B$ (and hence $q$ ) increases. In fact, the total moment saturates at the critical field $B_{c}$ to the value of

$$
M_{\|, \max }=\frac{e \rho_{s} L_{x}}{4 \hbar L_{\mathrm{d}}^{2}} L_{x} L_{y} d .
$$

The analysis above applies if the field and supercurrents are sufficiently small that their presence does not modify the pinning length $L_{\mathrm{d}}$, i.e., the supercurrents do not depin the vortices. This is the case if $q<1 / \xi$, the inverse disorder correlation length, so that the additional phase winding at wave vector $q$ in Eq. (5) does not affect the tunneling energy. For larger fields, however, the tunnel currents will oscillate within each correlation area, rapidly suppressing the net tunneling at larger scales. The current $j_{p}$, which is $j_{d}$ at the boundary, will then be approximately uniform, and $\boldsymbol{j}_{\mathrm{cf}}$ and $j_{t}$ will be small at long wavelengths. Thus we expect the susceptibility to fall quickly for fields beyond

$$
B_{c 2} \sim \frac{\hbar}{e d \xi} \sim 0.5 \mathrm{~T} .
$$

This can be regarded as a depinning field where the phase is no longer pinned by the random field but is determined by the diamagnetic wave vector $q$. A corresponding suppression ${ }^{28}$ of the current in interlayer tunneling experiments is expected, and observed, at such fields. Since $B_{c} / B_{c 2}=L_{x} \xi / 2 L_{\mathrm{d}}^{2}<1$ for experimental samples with $L_{x} \sim 1 \mathrm{~mm}$, the saturation at $B_{c}$ occurs before this suppression at $B_{c 2}$. Note that in the lattice model discussed above, the disorder is uncorrelated between 
sites, so that the numerical simulations do not capture the depinning effect at $B_{c 2}$.

\section{FLUX CREEP AND DISSIPATION AT LOW TEMPERATURES}

Let us now consider thermal fluctuations over the energy barriers which pin the winding of the superfluid phase. This allows the supercurrents to relax to the equilibrium state with $\boldsymbol{j}_{\text {cf }}=0$ and $j_{t}=0$. The diamagnetic moments induced by an in-plane field will decay as phase slips enter the system. This gives rise to resistivity in transport measurements. ${ }^{40}$ We will estimate the size of these effects, applying the conventional flux-creep model of superconductors. ${ }^{34}$ We neglect the possibility of vortex-glass states, ${ }^{41}$ which may further suppress the dissipation at low temperatures.

For sufficiently low temperatures $T$, the dynamics of the merons controlling the diamagnetic moment will involve thermal fluctuations of the phase domains of size $L_{\mathrm{d}}$ discussed above. As in the Anderson-Kim theory, ${ }^{34}$ uncorrelated vortex motion should be irrelevant at low temperatures and small bias, because the vortex separation $\xi \ll L_{\mathrm{d}}$, so the energy barriers are larger. A thermal fluctuation in which the phase of a domain changes by approximately $2 \pi$ corresponds to a phase slip moving a distance $L_{\mathrm{d}}$. This will occur at a frequency $\omega_{0} e^{-U(j) / k T}$, where $\omega_{0}$ is an attempt frequency and $U(j)$ is an energy barrier. Such phase slip dynamics implies an interlayer voltage according to the Josephson relation $V=\hbar \dot{\theta} / e$. For a typical domain size of $L_{\mathrm{d}}$, this gives a layer-antisymmetric in-plane electric field

$$
E=E_{\text {top }}-E_{\text {bottom }} \approx \frac{\hbar \omega_{0}}{e L_{\mathrm{d}}} e^{-U(j) / k T}
$$

At zero current, the typical energy barrier $U(j)$ will be the energy of a domain, which is the stiffness $\rho_{s}$ irrespective of $\operatorname{size}^{28}$ in two dimensions. The barrier will vanish at the current scale associated with the domain, $j_{c}=I_{\mathrm{d}} / L_{\mathrm{d}} \approx e \rho_{s} /\left(\hbar L_{\mathrm{d}}\right)$. The linear interpolation, $U(j)=\rho_{s}\left(1-j / j_{c}\right)$, corresponds to the Anderson-Kim model. Inserting this form in Eq. (18), we obtain for the Ohmic regime a sheet resistance for counterflow currents of

$$
R_{s} \approx\left(\hbar^{2} \omega_{0} / e^{2} k T\right) e^{-\rho_{s} / k T} \quad\left(k T \ll \rho_{s}\right) .
$$

The in-plane magnetic moment, and the long-wavelength counterflow supercurrent, relaxes in time $t$ as $^{42}$

$$
M_{\|}(t) / M_{\|, T=0} \approx 1-\left(k T / \rho_{s}\right) \ln \left(\omega_{0} t\right),
$$

where $M_{\|, T=0}$ is the $T=0$ moment from Eq. (15).

It is possible to extend this argument into the dissipative regime at high bias or temperature, where the barriers become irrelevant. The simplest assumption would be that the domain rotates every attempt time $1 / \omega_{0}$ when the current density is $j_{c}$. This gives a resistivity in the flux-flow regime of

$$
R_{s}^{*} \approx \frac{\hbar^{2} \omega_{0}}{e^{2} \rho_{s}}
$$

so that we can rewrite the flux-creep form as

$$
R_{s} \approx R_{s}^{*} e^{-\rho_{s} / k T}
$$

However, details of the dissipation are likely to change between these two regimes and other parameters may enter $R_{s}^{*}$, so that the prefactor in Eq. (22) will not be exactly the resistivity in the dissipative regime. Provided that $R_{s}^{*}$ is not fitted too far from the flux-creep regime, this is unimportant in practice because the exponential factor in general dominates in Eq. (22). Over a wider range of temperatures and currents, effects such as the activation of quasiparticles may appear, leading to additional exponential factors in the dissipation.

Reliable calculations of $\omega_{0}$ are very difficult, and irrelevant in practice in the flux-creep form, Eq. (22). Nonetheless, an estimate may be obtained by considering the dynamical equation for the phase, ${ }^{12,29}$

$$
\nabla^{2} \eta-\frac{1}{\lambda_{J}^{2}} \sin \left(\eta+\theta_{0}\right)=\frac{\hbar^{2}}{e^{2} \rho_{s}}\left[c \ddot{\eta}-\frac{1}{\rho_{z}} \dot{\eta}-\frac{1}{\rho_{x x}} \nabla^{2} \dot{\eta}\right],
$$

where $\rho_{x x}\left(\rho_{z}\right)$ is the resistivity of in-plane (tunneling) quasiparticle currents, and $c$ is the interlayer capacitance per unit area. This gives several candidates for the frequency scale at length scale $L_{\mathrm{d}}$. Since the resistivities are activated, we expect the first (inertial) term in Eq. (23) to control the dynamics at sufficiently low temperatures: $\omega_{0}=$ $\left(e / \hbar L_{\mathrm{d}}\right)\left(\rho_{s} / c\right)^{1 / 2}$.

\section{DISCUSSION}

We have examined the diamagnetic response of an isolated bilayer due to counterflow superfluidity. We obtained an inplane diamagnetic moment which saturates at a critical value $B_{c}$. Our theory also predicts a field scale $B_{c 2}$ for the suppression of the in-plane diamagnetic response. There would be no inplane susceptibility in a field-cooled bilayer, even below $B_{c 2}$, allowing a separation of the superfluid signal. We will now discuss briefly the magnitude and time scales of these effects using realistic parameters for current experiments.

From Eqs. (20) and (22), we see that the diamagnetic moment is long-lived, and the dissipation small, when $k T \ll$ $\rho_{s}$. In previous work, ${ }^{28}$ we estimated that $\rho_{s} \approx 20 \mathrm{mK}$ in current experiments. This small value arises from the finite interlayer separation and the reduced area of the sample containing superfluid in the coherence network picture. Using this estimate as well as a similar area reduction for the capacitance, we find for the attempt frequency $\omega_{0} \approx 300 \mathrm{MHz}$. Since the superfluid stiffness $\rho_{s}$ is around the lowest temperatures achieved, we expect from Eq. (20) that the moments relax rapidly. Similarly, counterflow dissipation due to flux motion would be significant with $R_{s}^{*} \approx 1-10 \Omega$.

Activated forms similar to Eq. (22) have previously been obtained for the residual counterflow resistivity based on hopping of in-plane vortices (merons) within the coherence network. ${ }^{30}$ In contrast, here we have obtained this form from the motion of phase-polarized domains, which corresponds to a large-scale collective motion of the merons. However, we expect the energy barriers for vortex motion in two dimensions 
to be the superfluid stiffness up to logarithmic factors, so long as the possibility of a vortex glass is excluded. Thus this form is not dependent on the precise model of vortex motion. We note that experiments on hole bilayers ${ }^{43}$ see this activated behavior in the counterflow resistance with a value of $20 \Omega$ at $30 \mathrm{mK}$ with an activated form that agrees with Eq. (19) if we use $\rho_{s} \approx 100 \mathrm{mK}$. Electron bilayers ${ }^{44}$ fit an approximately activated form with similar resistance values. A complete theory of dissipation and transport is beyond the scope of this work. Other mechanisms of dissipation will also play a role, such as the thermal excitation of vortices ${ }^{45}$ as well as fermionic quasiparticles with an energy gap of the order of $\rho_{s}$. It suffices to point out that the contribution from flux motion is not negligible in current samples. However, the activated form of Eq. (22) suggests that a modest increase in $\rho_{s}$, achievable by reducing the interlayer separation, would allow the study of the nearly dissipationless behavior of the flux-creep regime.

We now turn to the feasibility of measuring the diamagnetic response of a bilayer at low temperatures. To do this, we compare the maximum moment $M_{\|, \max }$ [Eq. (16)] with the scale of the perpendicular moment, $M_{\perp}=L_{x} L_{y} e \omega_{c} / 2$, for an electron gas in the integer quantum Hall regime with cyclotron energy $\hbar \omega_{c} \approx 50 \mathrm{~K}$ in this system. Using our previous estimates $\rho_{s} \approx 20 \mathrm{mK}$ and $L_{\mathrm{d}} \approx 100 \mu \mathrm{m}$, we obtain $M_{\|, \max } / M_{\perp}=$ $\left(\rho_{s} / 2 \hbar \omega_{c}\right)\left(L_{x} d / L_{\mathrm{d}}^{2}\right) \sim L_{x} /(2000 \mathrm{~m})$ when $d=28 \mathrm{~nm}$. This smallness of the effect, in comparison to the conventional magnetic moment, makes this very challenging to measure using torque magnetometry. This can also be inferred by noting that in our theory the critical currents in tunneling experiments are the maximum diamagnetic currents, and the former are experimentally in the nano-ampere range.

However, we can exploit our understanding of the disordered isolated bilayer to consider how this diamagnetic response can be increased. In particular, we note that $M_{\| \text {, max }}$ per unit volume increases with $L_{x}$ and with the number of polarized domains in the sample. Note that the interlayer tunneling can be increased by many orders of magnitude compared with the current samples by reducing the tunnel barrier. Increasing the tunneling strength reduces the domain size $L_{\mathrm{d}}$. Also, the narrower barriers possible with stronger tunneling allow a larger $\rho_{s}$. Our approach holds up to the point where the domain size $L_{\mathrm{d}}$ reaches the disorder length scale, $\xi \sim 100 \mathrm{~nm}$, and the maximum field $B_{c}$ just reaches the depinning field $B_{c 2}$. At this point, we obtain a significant magnetic moment $M_{\|, \max } / M_{\perp} \gtrsim$ $L_{x} /(1 \mathrm{~mm})$. Thus the diamagnetism of the exciton superfluid should be evident in samples with strong interlayer tunneling.

Although in the weak-tunneling samples the moments are small, the pinning and dynamics of the in-plane flux can still be probed in transport experiments. One consequence of the pinning picture is that the critical current in a tunneling experiment should not be affected by in-plane fields smaller than $B_{c 2}$, in contrast to the clean limit where the critical current is suppressed at fields ${ }^{37} B_{0}<B_{c 2}$. This is because in the pinning picture it is the gradients of the in-plane flux density which drive the flux through the disorder, and while the injected currents in the tunneling geometry impose such gradients, a uniform in-plane field does not. It would also be possible to study the dynamics of the in-plane flux in tunneling experiments. An interlayer voltage at one end of the sample introduces in-plane flux at a given rate, and measuring the interlayer voltages and currents reveals the subsequent motion ${ }^{42}$ of this flux. The logarithmic relaxation of the flux-creep regime can lead to hysteresis, which is seen in tunneling experiments. ${ }^{19}$

An interesting extension of our work would be to consider samples in the Corbino disk geometry. In such a geometry, a radial magnetic field could induce circulating counterflow supercurrents, similar to the induction of linear counterflow supercurrents by an in-plane field in the open geometry of Fig. 1. In the open geometry, tunneling is necessary to close the current loop, which we have seen gives rise to a maximum magnetic moment at the field $B_{c}$. In a Corbino disk, tunneling is not needed to close a circulating current loop, so there may be no saturation effect at $B_{c}$ and the full magnetic response may persist up to $B_{c 2}$. However, there will still be radial variations in the densities of applied flux and diamagnetic current, and maintaining the diamagnetic current loop requires that these variations are pinned. Thus it seems likely that, even in this geometry, there is a maximum magnetic moment determined by the sample dimensions and a pinning length. The relevant pinning length, however, may be determined by parameters other than the tunneling strength.

\section{CONCLUSIONS}

In summary, we have considered the diamagnetic response of a bilayer exciton superfluid to an in-plane magnetic field in the presence of in-plane vortices nucleated by charge disorder. We argue that at low temperatures, changes in the in-plane magnetic field induce circulating diamagnetic currents and hence persistent diamagnetic moments. The maximum moments which can be induced are determined by the pinning of the in-plane flux by the disorder, which involves a characteristic length scale related to the tunneling. At finite temperatures, thermal motion of the in-plane flux will lead to transport resistivities and cause the diamagnetic moments to decay. In current samples, we find that the maximum moments are small, but samples with stronger tunneling could allow persistent exciton supercurrents to be probed by torque magnetometry. Such experiments would be analogous to the torsional oscillator experiments that are the definitive measure of superfluid fraction in helium.

Finally, we note that very strong tunneling, or very large stiffness, may even be able to prevent the disorder nucleating vortices, ${ }^{31}$ recovering the clean limit in which the magnetic response is also expected to be measurable. ${ }^{24}$ In this case, the phase diagram of the bilayer would then closely parallel that of a type-II superconductor, with both a mixed state (as described here) and a clean state, experimentally distinguishable by the presence of hysteresis.

\section{ACKNOWLEDGMENTS}

We acknowledge support from Science Foundation Ireland (SFI/09/SIRG/I1592) (PRE) and EPSRC EP/F032773/1 (NRC), and we thank J. P. Eisenstein for helpful discussions. 
${ }^{1}$ A. J. Leggett, Rev. Mod. Phys. 71, S318 (1999).

${ }^{2}$ J. Blatt, K. Böer, and W. Brandt, Phys. Rev. 126, 1691 (1962).

${ }^{3}$ W. Kohn and D. Sherrington, Rev. Mod. Phys. 42, 1 (1970).

${ }^{4}$ E. Hanamura and H. Haug, Solid State Commun. 15, 1567 (1974).

${ }^{5}$ Y. E. Lozovik and V. I. Yudson, JETP Lett. 22, 274 (1975).

${ }^{6}$ A. V. Balatsky, Y. N. Joglekar, and P. B. Littlewood, Phys. Rev. Lett. 93, 266801 (2004).

${ }^{7}$ J.-J. Su and A. H. MacDonald, Nat. Phys. 4, 799 (2008).

${ }^{8}$ Y. M. Bunkov and G. E. Volovik, J. Phys.: Condens. Matter 22, 164210 (2010).

${ }^{9}$ M. Wouters and I. Carusotto, Phys. Rev. Lett. 105, 020602 (2010).

${ }^{10}$ J. P. Eisenstein and A. H. MacDonald, Nature (London) 432, 691 (2004).

${ }^{11}$ H. A. Fertig, Phys. Rev. B 40, 1087 (1989).

${ }^{12}$ X. G. Wen and A. Zee, Phys. Rev. B 47, 2265 (1993).

${ }^{13}$ Z. F. Ezawa and A. Iwazaki, Phys. Rev. B 48, 15189 (1993).

${ }^{14}$ S. Q. Murphy, J. P. Eisenstein, G. S. Boebinger, L. N. Pfeiffer, and K. W. West, Phys. Rev. Lett. 72, 728 (1994).

${ }^{15}$ T. S. Lay, Y. W. Suen, H. C. Manoharan, X. Ying, M. B. Santos, and M. Shayegan, Phys. Rev. B 50, 17725 (1994).

${ }^{16}$ I. B. Spielman, J. P. Eisenstein, L. N. Pfeiffer, and K. W. West, Phys. Rev. Lett. 84, 5808 (2000).

${ }^{17}$ A. D. K. Finck, A. R. Champagne, J. P. Eisenstein, L. N. Pfeiffer, and K. W. West, Phys. Rev. B 78, 075302 (2008).

${ }^{18}$ Y. Yoon, L. Tiemann, S. Schmult, W. Dietsche, K. von Klitzing, and W. Wegscheider, Phys. Rev. Lett. 104, 116802 (2010).

${ }^{19}$ L. Tiemann, Y. Yoon, W. Dietsche, K. von Klitzing, and W. Wegscheider, Phys. Rev. B 80, 165120 (2009).

${ }^{20}$ L. Tiemann, W. Dietsche, M. Hauser, and K. von Klitzing, New J. Phys. 10, 045018 (2008).

${ }^{21}$ D. W. Snoke, Adv. Condens. Matter Phys. 2011, 938609 (2011).

${ }^{22}$ J.-J. Su and A. H. MacDonald, Phys. Rev. B 81, 184523 (2010).
${ }^{23}$ D. A. Pesin and A. H. MacDonald, Phys. Rev. B 84, 075308 (2011).

${ }^{24}$ C. B. Hanna, A. H. MacDonald, and S. M. Girvin, Phys. Rev. B 63, 125305 (2001).

${ }^{25}$ K. Yang, K. Moon, L. Zheng, A. H. MacDonald, S. M. Girvin, D. Yoshioka, and S.-C. Zhang, Phys. Rev. Lett. 72, 732 (1994).

${ }^{26}$ S. I. Shevchenko, Phys. Rev. B 56, 10355 (1997).

${ }^{27}$ D. K. K. Lee, P. R. Eastham, and N. R. Cooper, Adv. Condens. Matter Phys. 2011, 792125 (2011).

${ }^{28}$ P. R. Eastham, N. R. Cooper, and D. K. K. Lee, Phys. Rev. Lett. 105, 236805 (2010).

${ }^{29}$ M. M. Fogler and F. Wilczek, Phys. Rev. Lett. 86, 1833 (2001).

${ }^{30}$ H. A. Fertig and G. Murthy, Phys. Rev. Lett. 95, 156802 (2005).

${ }^{31}$ P. R. Eastham, N. R. Cooper, and D. K. K. Lee, Phys. Rev. B 80, 045302 (2009).

${ }^{32}$ A. Stern, S. M. Girvin, A. H. MacDonald, and N. Ma, Phys. Rev. Lett. 86, 1829 (2001).

${ }^{33}$ L. Balents and L. Radzihovsky, Phys. Rev. Lett. 86, 1825 (2001).

${ }^{34}$ P. W. Anderson and Y. B. Kim, Rev. Mod. Phys. 36, 39 (1964).

${ }^{35}$ L. Rademaker, J. Zaanen, and H. Hilgenkamp, Phys. Rev. B 83, 012504 (2011)

${ }^{36}$ P. Bak, Rep. Prog. Phys. 45, 587 (1982).

${ }^{37}$ D. V. Fil, Phys. Rev. B 82, 193303 (2010).

${ }^{38}$ N. Read, Phys. Rev. B 52, 1926 (1995).

${ }^{39} \mathrm{M}$. Tinkham, Introduction to Superconductivity, 2nd ed. (McGrawHill, New York, 1996).

${ }^{40}$ D. A. Huse, Phys. Rev. B 72, 064514 (2005).

${ }^{41}$ T. Nattermann and S. Scheidl, Adv. Phys. 49, 607 (2000).

${ }^{42}$ A. Gurevich and H. Küpfer, Phys. Rev. B 48, 6477 (1993).

${ }^{43}$ E. Tutuc, M. Shayegan, and D. A. Huse, Phys. Rev. Lett. 93, 036802 (2004).

${ }^{44}$ M. Kellogg, J. P. Eisenstein, L. N. Pfeiffer, and K. W. West, Phys. Rev. Lett. 93, 036801 (2004).

${ }^{45}$ T. Hyart and B. Rosenow, Phys. Rev. B 83, 155315 (2011). 